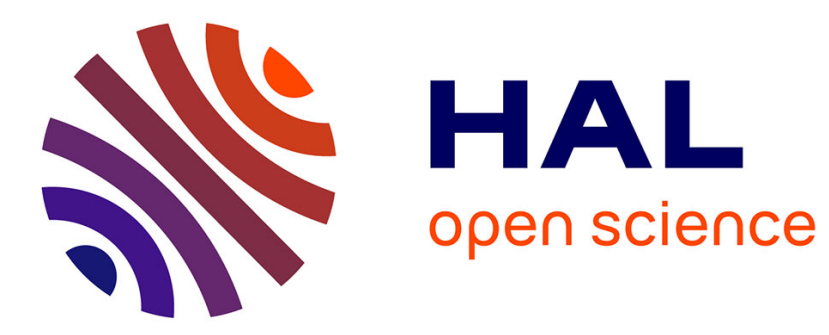

\title{
Diophantine conditions and real or complex Brjuno functions
}

\author{
P. Moussa, S. Marmi
}

\section{To cite this version:}

P. Moussa, S. Marmi. Diophantine conditions and real or complex Brjuno functions. Michel Planat. Noise, Oscillators and Algebraic Randomness. From Noise in Communication Systems to Number Theory. Lectures of a School Held in Chapelle des Bois, France, April 5-10, 1999, 550, Springer, pp.324-342, 2000, Lecture Notes in Physics, 978-3-540-67572-3. 10.1007/3-540-45463-2_16 . hal00087742

\section{HAL Id: hal-00087742 \\ https://hal.science/hal-00087742}

Submitted on 4 Sep 2020

HAL is a multi-disciplinary open access archive for the deposit and dissemination of scientific research documents, whether they are published or not. The documents may come from teaching and research institutions in France or abroad, or from public or private research centers.
L'archive ouverte pluridisciplinaire HAL, est destinée au dépôt et à la diffusion de documents scientifiques de niveau recherche, publiés ou non, émanant des établissements d'enseignement et de recherche français ou étrangers, des laboratoires publics ou privés. 


\title{
Diophantine Conditions and Real or Complex Brjuno Functions
}

\author{
Pierre Moussa ${ }^{1}$ and Stefano Marmi ${ }^{2}$ \\ 1 Service de Physique Théorique, CEA/Saclay, \\ F-91191 Gif sur Yvette cedex, France \\ 2 Dipartimento di Matematica "U.Dini" Università di Firenze, \\ Viale Morgagni 67/A, I-50134 Firenze, Italy
}

\begin{abstract}
The continued fraction expansion of the real number $x=a_{0}+x_{0}$, $a_{0} \in \mathbb{Z}$, is given by $0 \leq x_{n}<1, x_{n}^{-1}=a_{n+1}+x_{n+1}, a_{n+1} \in \mathbb{N}$, for $n \geq 0$. The Brjuno function is then $B(x)=\sum_{n=0}^{\infty} x_{0} x_{1} \ldots x_{n-1} \ln \left(x_{n}^{-1}\right)$, and the number $x$ satisfies the Brjuno diophantine condition whenever $B(x)$ is bounded. Invariant circles under a complex rotation persist when the map is analytically perturbed, if and only if the rotation number satisfies the Brjuno condition, and the same holds for invariant circles in the semi-standard and standard maps cases. In this lecture, we will review some properties of the Brjuno function, and give some generalisations related to familiar diophantine conditions. The Brjuno function is highly singular and takes value $+\infty$ on a dense set including rationals. We present a regularisation leading to a complex function holomorphic in the upper half plane. Its imaginary part tends to the Brjuno function on the real axis, the real part remaining bounded, and we also indicate its transformation under the modular group.
\end{abstract}

\section{Hamiltonian Chaos and the Standard Map}

The simplest non trivial model for Hamiltonian chaos is a two dimensional real map, called the "Standard Map". It has been introduced more or less independently by Chirikov and Taylor [1. [2. 2]. The occurrence of chaos was discussed by Greene [3] who displayed many numerical results on this model, which describes a simplified version of the non-linear coupling of two oscillators. It occurs naturally in many domains of physics, including celestial mechanics, classical quasiperiodic systems, quantum quasicrystals, adiabatic response in non-linear mechanics, magnetic toroidal configurations in plasma physics, non-linear electronic devices, and many others.

The Standard Map is a map from the cylinder $\mathbb{T} \times \mathbb{R}$ to itself, defined as

$$
(\theta, r) \mapsto\left(\theta^{\prime}, r^{\prime}\right)=\left(\theta+r+\frac{K}{2 \pi} \sin (2 \pi \theta)(\bmod 1), r+\frac{K}{2 \pi} \sin (2 \pi \theta)\right) .
$$

Note that the second variable can also be taken modulo 1 , in which case we get a map $\mathbb{T}^{2} \rightarrow \mathbb{T}^{2}$. The above map can be written in two equivalent forms:

- Hamiltonian form

$$
r^{\prime}-r=\frac{K}{2 \pi} \sin (2 \pi \theta) \quad, \quad \theta^{\prime}-\theta=r^{\prime},
$$


- Lagrangian form, where one considers now the twice iterated map, that is $(\theta, r) \rightarrow\left(\theta^{\prime}, r^{\prime}\right) \rightarrow\left(\theta^{\prime \prime}, r^{\prime \prime}\right)$, so that

$$
\theta^{\prime \prime}-2 \theta^{\prime}+\theta=\frac{K}{2 \pi} \sin \left(2 \pi \theta^{\prime}\right)
$$

and for the $n$-th iterated map one gets

$$
\theta_{n+1}-2 \theta_{n}+\theta_{n-1}=\frac{K}{2 \pi} \sin \left(2 \pi \theta_{n}\right) .
$$

This last equation is sometimes called the Frenkel-Kontorova model 四 which describes equilibrium positions of a chain of material points placed in a periodic potential and submitted to an harmonic elastic force between two neighbouring points.

If $K=0$, we get the so-called "twist-map", which gives

$$
r_{n+1}=r_{n}=r_{0}=\rho=\mathrm{constant} \quad, \quad \theta_{n+1}=\theta_{0}+n \rho(\bmod 1),
$$

after $n$ iterations. This map is nothing but a rotation of angle $2 \pi \rho$-we say that the rotation number is $\rho$. The orbits are all transverse to the axis of the cylinder. They are made of a finite number of points (and therefore discrete) if $\rho$ is rational, and they are dense on transverse circles if $\rho$ is irrational. The cylinder is sliced along orbits at irrational values of $r$, which are intertwined with discrete orbits at rational values of $r$. The question is : in which sense is such a pattern stable under perturbations, that is here when $K \neq 0$ ?

Among the orbits which are dense in a curve wrapped around the cylinder, particularly interesting are the orbits which will persist under perturbation, in particular because they separate the space into domains which do not communicate. It is known that when $K$ is large (for example $K>4 / 3$, see [5]), such orbits do not exist, and on the other hand, when $K$ is small, some of the irrational orbits persist, depending on arithmetical properties of the rotation number. For perturbed twist maps, we define the rotation number as $\lim _{n \rightarrow \infty} n^{-1} \theta_{n}$, where $\left(\theta_{n}, r_{n}\right)$ is the $n$-th iterated map obtained from (1).

Other kinds of invariant curves may occur, attached to elliptic periodic orbits. For instance if $K>0$ is sufficiently small, the point $(1 / 2,0)$ is an elliptic fixed point. Due to KAM Theorem (see [6] for a review), there exist homotopically trivial invariant curves on the cylinder winding around this fixed point, which form the so-called elliptic islands. We shall not consider here the problem of such orbits, although there existence is very important in connection with ergodic theory. Indeed one expects chaotic behaviour for $K$ large, but the persistence of elliptic islands could prevent the map from being ergodic.

\section{The Critical Constants}

For the standard map, we consider now the homotopically non-trivial invariant curves i.e. wrapped around the cylinder. A natural way to look for their 
existence is to replace the angular variable $\theta$ by the new variable $\phi \in \mathbf{T}$

$$
\theta=\phi+u(\phi, K, \rho) \quad, \quad r=\rho+u(\phi, K, \rho)-u(\phi-\rho, K, \rho) .
$$

With the condition $1+\partial u / \partial \phi>0$, it would describe a curve around the cylinder, on which the map is expressed as $\phi^{\prime}=\phi+\rho$, when $\phi$ describes $\mathbf{T}$ for $K$ and $\rho$ fixed. We say that (6) expresses on the curve the conjugacy of the map to a rotation. The existence of a function $u(\phi, K, \rho)$, analytic in the variable $\phi$, insures the existence of an analytic invariant curve with rotation number $\rho$. We are interested to determine the critical constant $K_{c}(\rho)$ as being the largest possible value of $K$ for which such an analytic function $u$ exists. Of course, one could consider regularity constraints weaker than analyticity, leading to other critical constants. We look for a perturbation expansion of the function $u$, and we follow the notations of [7]. From the standard map we get from (6)

$$
u(\phi+\rho, K, \rho)-2 u(\phi, K, \rho)+u(\phi-\rho, K, \rho)=\frac{K}{2 \pi} \sin (2 \pi(\phi+u(\phi, K, \rho)) .
$$

For $k \geq 1$, we have

$u^{(k)}(\phi+\rho, \rho)-2 u^{(k)}(\phi, \rho)+u^{(k)}(\phi-\rho, \rho)=\left.\frac{1}{2 \pi} \sin (2 \pi \phi+2 \pi u(\phi, K, \rho))\right|_{k-1}$,

where in the right hand side, one keeps only the terms of order $k-1$ in the expansion on powers of $K$. We use now the Fourier series expansion on $\phi$, that is $u^{(k)}(\phi, \rho)=\sum_{\nu \in \mathbf{Z}} u_{\nu}^{(k)}(\rho) e^{2 i \pi \nu \phi}$, and we see that the coefficient of $e^{2 i \pi \nu \phi}$ in the left hand side of $(8)$ is $2(\cos (2 \pi \nu \rho)-1) u_{\nu}^{(k)}(\rho)$. Therefore (8) allows a recursive computation of the Fourier coefficients $u_{\nu}^{(k)}(\rho)$, and we get for $u^{(k)}(\phi, \rho)$ expressions as trigonometric polynomials in $\phi$. However terms of the kind $2(\cos (2 \pi \nu \rho)-1)$ occur in the denominators along the steps of the recursion. Such factors are called "small divisors", some of them vanish when $\rho$ is rational, and may become arbitrarily small when $\nu$ becomes large, for irrational $\rho$. Now let $\widetilde{K}_{c}(\rho)$ be the minimum over $\phi$ of the convergence radius of the expansion

$$
u(\phi, K, \rho)=\sum_{k=1}^{\infty} K^{k} u^{(k)}(\phi, \rho)
$$

For $\rho$ rational, (8) cannot be solved, and we set $\widetilde{K}_{c}(\rho)=0$. For irrational values of $\rho$, Berretti and Gentile [8] were able to control $\widetilde{K}_{c}(\rho)$ using the Brjuno function $B(\rho)$ which is a number theoretic function which will define in the following Section 4. More precisely, there exists $C>0$ such that, for any irrational $\rho$,

$$
\left|\ln \left(\left(\widetilde{K}_{c}(\rho)\right)^{-1}\right)-2 B(\rho)\right|<C .
$$


The functions $\widetilde{K}_{c}(\rho)$ and $e^{-2 B(\rho)}$ both vanish on all rationals, but the previous equation shows that the ratio $\widetilde{K}_{c}(\rho) / e^{-2 B(\rho)}$ remains uniformly bounded at every irrationals. The fact that this ratio remains bounded is in itself amazing, but it recalls earlier and now classical results by Yoccoz [9] on the linearisation of holomorphic maps. We shall see later that we may have even better results in the framework of holormorphic maps.

The determination of the radius of convergence in (9) is not the whole story. It is possible that the function $u$ may be analytically continued for real values of $\phi$ and for $K>\widetilde{K}_{c}(\rho)$ real. Thus we would have another critical constant $K_{c}(\rho)$ such that we still have real analytic curves for $\widetilde{K}_{c}(\rho)<K<$ $K_{c}(\rho)$ real. The numerical results 10 seem to indicate that this is indeed the case: see 11] for a detailed discussion of this issue both from the numerical and the analytical points of view, which also uses results of [12,13]. The definitive answer is not known to us today, although we are led to expect that the function $B(\rho)$ plays a central role in the determination of $K_{c}(\rho)$ (see also Davie [14).

\section{Complex Analytic Maps}

The problems of the critical constant is better understood in the case of the complex analytic maps. We have already seen in (10) that the critical constant $\widetilde{K}_{c}(\rho)$ of the complexified version of the standard map is controlled by the Brjuno function. A simpler example is the "Semi-standard Map", which is a two dimensional complex map on the cylinder, closely related to the standard map (1) : to get the semi-standard map, just replace in (1) the sine function $\sin (2 \pi \theta)$ by its positive frequency part $(1 / 2 i) \exp (2 i \pi \theta)$. The procedure to get analytic invariant curves proceeds in a completely similar way as Equations (6) to (9), and it was proven that in this case [15, 16, the critical constant $K_{\text {ssm }}(\rho)$ defined in a same way as above, fulfils as in (10)

$$
\left|\ln \left(\left(K_{\mathrm{ssm}}(\rho)\right)^{-1}\right)-2 B(\rho)\right|<C .
$$

The numerical results (especially the figure 16) in ref. [16] provide more. Not only the ratio $\left.K_{\mathrm{ssm}}(\rho)\right) / e^{-2 B(\rho)}$ is bounded on irrationals, but it is extendable to a continuous function on $[0,1]$, bounded below and above by positive constants. This result is amazing if one remember that both $\left.K_{\mathrm{ssm}}(\rho)\right)$ and $e^{-2 B(\rho)}$ vanish at all rationals. Therefore the Brjuno function $B(\rho)$ is a good model to represent the singular behavior of $\ln \left(\left(K_{\mathrm{ssm}}(\rho)\right)^{-1}\right)$.

The Brjuno function was introduced by Yoccoz [9] in the apparently simpler problem of the linearisation of complex holomorphic maps around their fixed points. This is a more than one century old problem (see [17] for a nice review), which we can state as follows. Let $f(z)$ be a holomorphic map such that $f(0)=0, f^{\prime}(0)=e^{2 i \pi \rho}$. Is it possible to conjugate the map $f$ to its linear part? This means that we look for a function $h$, holomorphic in a disk 
of radius $R_{f}$, such that $h(0)=0, h^{\prime}(0)=1$, and $f(h(z))=h\left(z e^{2 i \pi \rho}\right)$. Note that such a function $h$, if it exists, is unique. In this case, the function $f$ is said to admit a Siegel disk of radius $R_{f}$. The Siegel disk is a topological disk with conformal radius $R_{f}$, since it is the image through the normalised conformal map $h$ of the disk $|z|<R_{f}$.

We quote now the classical results on this question 17]. i) If $\rho$ is rational, there is no disk, that is $R_{f}=0$. ii) if $\rho$ is irrational and satisfies a (strong) Liouville condition, we still have $R_{f}=0$. iii) if $\rho$ is a diophantine irrational (see Section 5 below), then there exists a Siegel disk and $R_{f}>0$, more precisely this happens when $B(\rho)$ is finite. iv) If $B(\rho)=+\infty$, then that there exist functions $f$ such that $R_{f}=0$. Indeed Yoccoz [9] proved the following : define $R(\rho)$ as the smallest radius of the Siegel disks $R_{f}$ obtained when $f$ varies in the compact family of all univalent maps on the unit disk such that $f(0)=0$, and $f^{\prime}(0)=e^{2 i \pi \rho}$. Then we have

$$
\left|\ln \left((R(\rho))^{-1}\right)-B(\rho)\right|<C .
$$

Now consider the family of quadratic polynomial $P_{2}(z)=e^{2 i \pi \rho}\left(z-z^{2}\right)$, and call $R_{2}(\rho)$ the radius of the Siegel disk associated to it. Observe first that, through the rescaling $z \rightarrow e^{-2 i \pi \rho} R \times z$, then $P_{2}$ is transformed in $e^{2 i \pi \rho} z-R z^{2}$. In the rescaled variable, we see that $R_{2}$ is the maximum value of the constant $R$ for which a circle with conformal radius one is persistant. Therefore $R_{2}(\rho)$ is the critical constant adapted to the present case, and this leads to the analogies between (10), or its equivalent in the real case, and (12).

Here again, the numerical results (now the figure 6) in ref. [16] bring some continuity properties. Not only the ratio $\left.R_{2}(\rho)\right) / e^{-B(\rho)}$ is bounded on irrationals, but it is extendable to a continuous function on $[0,1]$, bounded below and above by positive constants. Therefore the Brjuno function $B(\rho)$ is again a good model to represent the singular behavior of $\ln \left(\left(R_{2}(\rho)\right)^{-1}\right)$. In our work [18] which started from these observations, we give arguments which strongly support the conjecture that the ratio $\left.R_{2}(\rho)\right) / e^{-B(\rho)}$ is not only continuous but satifies a Hölder continuity condition with exponent $1 / 2$. More precisely, the Brjuno function displays the universal singular behaviour (up to some Hölder- $\frac{1}{2}$ continuous function) of the critical functions occuring in small divisors holomorphic problems in dimension one.

The use of the Brjuno function was somewhat implicit in the work of Buric et al. [19], where they attempted to find representations of the critical constants by what they called modular smoothing. Singular functions of the same type occured in MacKay [20] in relation to the Brjuno condition.

It is nevertheless useful to recall here briefly one of the steps, called renormalisation, which plays a special role in Yoccoz's argument, and will appear to be crucial in understanding the fine regularity properties of ratios of the type $\left.R_{2}(\rho)\right) / e^{-B(\rho)}$. For this purpose, we follow [17], and we we consider first a rotation of angle $2 \pi \rho$, with $0 \leq \rho<1$, that is $z \rightarrow e^{2 i \pi \rho} z$, acting in an open disk of radius $R_{\rho}$ centered at the origin in the complex plane. We need an arbitrary point $a$, such that $|a|=R_{\rho}$, and for 
simplicity we take the real point $a=R_{\rho}$. Let $a^{\prime}=e^{2 i \pi \rho} R_{\rho}$ its image. Now, consider the angular sector bounded by the lines $0 a$ and $0 a^{\prime}$, namely $\Delta_{\rho}=\left\{z|0 \leq \arg z<2 \pi \rho| z \mid,<R_{\rho}\right\}$, the line $0 a^{\prime}$ being excluded. Consider the orbit made of the successive iterated points $z_{n}, n \geq 1$ starting from $z_{0} \in \Delta_{\rho}$, and let $z_{q}$ the first of these points which also belong to $\Delta_{\rho}$. The map $z_{0} \rightarrow z_{q}$ is thus the first return map in the sector. We have $(2 \pi)^{-1}\left(\arg z_{q}\right)=(2 \pi)^{-1}\left(\arg z_{0}\right)+q \rho-1$. We now take in the sector the variable $u$ such that its complex conjugate $\bar{u}=z^{\frac{1}{\rho}}$, where now $u$ belongs to a disk of radius $R_{\rho}^{\frac{1}{\rho}}$. For the values $u_{0}$ and $u_{q}$, corresponding to $z_{0}$ and $z_{q}$, we have $(2 \pi)^{-1}\left(\arg u_{q}\right)=(2 \pi)^{-1}\left(\arg u_{0}\right)-q+\frac{1}{\rho}(\bmod 1)=(2 \pi)^{-1}\left(\arg u_{0}\right)+\frac{1}{\rho}$. The original map which acted in a disk of radius $R_{\rho}$, leads in the new "renormalised variable" $u$, to a rotation with rotation number $\frac{1}{\rho}$, acting in a disk with radius $R_{(1 / \rho)}$, such that $\ln R_{\rho}=\rho \ln R_{(1 / \rho)}$.

This construction extends to the non linear perturbed case, for example $P_{2}(\rho, z)=e^{2 i \pi \rho}\left(z-z^{2}\right)$, with a lot of complications. Suppose that there is a Siegel disk for $P_{2}(\rho, z)$. In this disk, there are conformal coordinates on which the maps is exactly a rotation of angle $2 \pi \rho$, and on these coordinates we apply the linear renormalisation. The problem is then to give an interpretation of the renormalised coordinates $u$ which we obtain. It appears that there exist a holomorphic map in the variable $u$ with rotation number $\rho^{-1}$, which admits a Siegel disk, with conformal radius $\widetilde{R}_{(1 / \rho)}$ such that $\ln R_{\rho}-\rho \ln \widetilde{R}_{(1 / \rho)}=0$. However, this map is not a polynomial with degree 2 . This led Yoccoz to extend the problem to the compact family of univalent map on the unit disk with rotation number $\rho$, and he has considered the minimum $R(\rho)$ of the radius of the Siegel disk taken over this family of maps. The result is two modifications to the relation $\ln R_{\rho}-\rho \ln R_{(1 / \rho)}=0$ obtained in the linear case. First due to the minimisation procedure, the best one could get is a positive uniform upper bound for this expression instead of zero. Second, there is a special difficulty when $\rho$ goes to zero. In this case the Siegel disk is strongly distorted, since there is an other fixed point which tends to zero when $\rho$ goes to zero. The comparison between the linear and the non linear case becomes unjustified in this limit. Yoccoz proved that the result is an additional logarithmic term in the estimate, so that we only get that $\ln R_{\rho}-\rho \ln R_{(1 / \rho)}-\ln \rho$ is bounded. It is therefore natural to compare the function $-\ln R(\rho)$ (as well as $\left.-\ln R_{2}(\rho)\right)$ to the solution of the equation $B(\rho)-\rho \ln B(1 / \rho)+\ln \rho=0$ which we will see, is nothing else than the Brjuno function.

\section{Continued Fractions and the Brjuno Function}

We first give a somewhat unusual definition of the continued fraction expansion sometimes called "japanese continued fractions" 211. Let $\alpha$ be a fixed

real number such that $\frac{1}{2} \leq \alpha \leq 1$. Then, given the starting number $x$, the coefficients $a_{n}$ and $\varepsilon_{n}$ are recursively uniquely defined by the conditions

$$
x=a_{0}+\varepsilon_{0} x_{0}, \text { and } \forall n \geq 0, x_{n}^{-1}=a_{n+1}+\varepsilon_{n+1} x_{n+1},
$$


with $\forall n \geq 0, \alpha-1 \leq \varepsilon_{n} x_{n}<\alpha$. We define the modified integer part $[x]_{\alpha}$ and the modified fractional part $\{x\}_{\alpha}$ as follows,

$$
[x]_{\alpha}=[x-\alpha+1]_{1} \quad \text { and } \quad\{x\}_{\alpha}=\{x-\alpha+1\}_{1}+\alpha-1,
$$

where $[x]_{1}$ and $\{x\}_{1}$ are the usual integer and fractional parts of $x$ (so that $\left.0 \leq\{x\}_{1}<1\right)$. With these notations, we can rewrite (13) as

$$
a_{0}=[x]_{\alpha}, \varepsilon_{0} x_{0}=\{x\}_{\alpha} \text {, and, } a_{n+1}=\left[x_{n}^{-1}\right]_{\alpha}, \varepsilon_{n+1} x_{n+1}=\left\{x_{n}^{-1}\right\}_{\alpha}, \forall n>0 \text {. }
$$

Therefore the $x_{n}$ are generated by iterating the function $A_{\alpha}(x)=\left|\left\{x^{-1}\right\}_{\alpha}\right|$, that is $\forall n \geq 0, \quad x_{n+1}=A_{\alpha}\left(x_{n}\right)=\left|\left\{x_{n}^{-1}\right\}_{\alpha}\right|=\left|x_{n}^{-1}-\left[x_{n}^{-1}\right]_{\alpha}\right|$. A more detailed description states that the map $A_{\alpha}$ is made of the following branches

$$
\begin{array}{ll}
\operatorname{branch} k^{+}: & A_{\alpha}(x)=\frac{1}{x}-k \quad \text { for } \quad \frac{1}{k+\alpha}<x \leq \frac{1}{k}, \\
\text { branch } k^{-}: & A_{\alpha}(x)=k-\frac{1}{x} \quad \text { for } \quad \frac{1}{k}<x \leq \frac{1}{k+\alpha-1} .
\end{array}
$$

When $\frac{1}{2}<\alpha \leq 1$, the function $A_{\alpha}$ maps the interval $[0, \alpha)$ to itself, whereas when $\alpha=\frac{1}{2}$, it maps the interval $[0, \alpha]$ to itself. In both cases, it is convenient to set $A_{\alpha}(0)=0$, and we get a map which is infinitely differentiable by pieces, and the points where it is not differentiable accumulate to 0 . Now $x$ and the reduced fraction $p_{n} / q_{n}$ admit the following representation

$$
x=a_{0}+\frac{\varepsilon_{0}}{a_{1}+\frac{\varepsilon_{1}}{a_{2}+\ddots+\frac{\varepsilon_{n-1}}{a_{n}+\varepsilon_{n} x_{n}}}} \quad, \quad \frac{p_{n}}{q_{n}}=a_{0}+\frac{\varepsilon_{0}}{a_{1}+\frac{\varepsilon_{1}}{a_{2}+\ddots+\frac{\varepsilon_{n-1}}{a_{n}}}} .
$$

As long as the $x_{n}$ 's do not vanish, we have

$$
x=\frac{p_{n}+p_{n-1} \varepsilon_{n} x_{n}}{q_{n}+q_{n-1} \varepsilon_{n} x_{n}} \quad, \quad x_{n}=\left(-\varepsilon_{n}\right) \frac{p_{n}-x q_{n}}{p_{n-1}-x q_{n-1}},
$$

and the recursion relations

$$
\begin{aligned}
& p_{n}=a_{n} p_{n-1}+\varepsilon_{n-1} q_{n-2}, p_{0}=a_{0}, p_{-1}=1, \\
& q_{n}=a_{n} q_{n-1}+\varepsilon_{n-1} q_{n-2}, q_{0}=1, q_{-1}=0
\end{aligned}
$$

so that we get $0<q_{0} \leq q_{1}<q_{2}<\ldots<q_{n}<q_{n+1}<\ldots$. We also define

$$
\beta_{n}=x_{0} x_{1} \cdots x_{n}=(-1)^{n} \varepsilon_{0} \varepsilon_{1} \cdots \varepsilon_{n}\left(q_{n} x-p_{n}\right),
$$

and we have

$$
\frac{1}{1+\alpha} \leq \beta_{n} q_{n+1}=\frac{q_{n+1}}{q_{n+1}+\varepsilon_{n+1} q_{n} x_{n+1}} \leq \frac{1}{\alpha} .
$$


Now there exist $\lambda(\alpha)$, with $0<\lambda(\alpha)<1$, and positive constants $C_{1}$ and $C_{2}$, such that 18

$$
\beta_{n}<C_{1} \lambda(\alpha)^{n} \quad, \quad q_{n}>C_{2} \lambda(\alpha)^{-n} .
$$

Indeed we have

$$
\begin{aligned}
& \text { for } \quad \frac{\sqrt{5}-1}{2}<\alpha \leq 1, \quad \lambda(\alpha)=\lambda(1)=\frac{\sqrt{5}-1}{2}=0.618 \ldots \\
& \text { and for } \frac{1}{2} \leq \alpha \leq \frac{\sqrt{5}-1}{2}, \lambda(\alpha)=\lambda\left(\frac{1}{2}\right)=\sqrt{2}-1=0.414 \ldots .
\end{aligned}
$$

When $x_{n}=0$ for some $n$, and $x_{m} \neq 0$ for $m<n$, then we have $x=p_{n} / q_{n}$ which is rational, and we say that the fraction stops at order $n$ (with our conventions, we have $x_{m}=0, \forall n \geq m$ ). Conversely, if $x$ is rational, the continued fraction expansion stops at some finite order $n$. For $\alpha=1$, we get the classical Gauss continued fraction expansion for which all signs $\varepsilon_{n}=+1$, and for $\alpha=1 / 2$, we have the continued fraction to the nearest integer. Note that when $\alpha \neq 1$, the results of equations (23a) and (23b) are not obvious. For details, and in particular for the extension to others values of $\alpha$, with $0 \leq \alpha<\frac{1}{2}$, see 18,22.

Given a positive real function $f$ on $(0,1)$, the Brjuno series $B_{f}^{(\alpha)}(x)$ is the sum (which can be infinite) of the series with positive terms

$B_{f}^{(\alpha)}(x)=\sum_{n=0}^{\infty} \beta_{n-1} f\left(x_{n}\right)=f\left(x_{0}\right)+x_{0} f\left(x_{1}\right)+\ldots+x_{0} x_{1} \cdots x_{n-1} f\left(x_{n}\right)+\ldots$,

where $\frac{1}{2} \leq \alpha \leq 1$, and for $k \geq 0, x_{k}$ defined in Eq. (13) or (15). As mentioned above, when $x$ is rational, we have $x_{n}=0$ for some $n$, and we use $x_{m}=0$ for $m \geq n$, The following results are easily obtained from the definitions

$$
\begin{aligned}
& B_{f}^{(\alpha)}(x)=B_{f}^{(\alpha)}(x+1), \\
& B_{f}^{(\alpha)}(x)=x B_{f}^{(\alpha)}\left(\frac{1}{x}\right)+f(x) \text { for } 0<x<\alpha, \\
& B_{f}^{(\alpha)}(x)=B_{f}^{(\alpha)}(-x) \text { for } 0<x \leq 1-\alpha .
\end{aligned}
$$

In particular, $B_{f}^{\left(\frac{1}{2}\right)}(x)$ is an even function. More surprising is the following result [18]: in the $\alpha=1$ case, for $B_{f}^{( \pm)}(x)=\frac{1}{2}\left(B_{f}^{(1)}(x) \pm B_{f}^{(1)}(-x)\right)$ which are the even and odd parts of $B_{f}^{(1)}(x)$, we have for $0<x \leq \frac{1}{2}$,

$$
\begin{aligned}
B_{f}^{(-)}(x) & =\frac{1}{2}\left(f(x)-f(1-x)-(1-x) f\left(\frac{x}{1-x}\right)\right), \\
B_{f}^{(+)}(x) & =x B_{f}^{(+)}\left(\frac{1}{x}\right)+\frac{1}{2} G(x), \quad \text { with } \\
G(x) & =f(x)+f(1-x)+(1-x) f\left(\frac{x}{1-x}\right)+2 x B_{f}^{(-)}\left(\frac{1}{x}\right) .
\end{aligned}
$$


In order to prove the previous equations, we use Equations $(23 \mathrm{a}-\mathrm{c})$ and the succession of transformations

$$
-x \rightarrow 1-x \rightarrow \frac{1}{1-x} \rightarrow \frac{1}{1-x}-1=\frac{x}{1-x} \rightarrow \frac{1-x}{x}=\frac{1}{x}-1 \rightarrow \frac{1}{x} \rightarrow x,
$$

which provides the requested relations between $B(x)$ and $B(-x)$.

Now, it is convenient to introduce the following specific notations:

i) In $B_{f}^{(\alpha)}(\mathrm{x})$, when $\alpha=1$, we omit the superscript $(\alpha)$, and when $\alpha=\frac{1}{2}$, we replace the superscript $(\alpha)$ by $e$, so that $B_{f}(x)=B_{f}^{(1)}(x)$ and $B_{f}^{e}(x)=$ $B_{f}^{(1 / 2)}(x)$ respectively.

ii) We omit the subscript $f$ when $f(x)=-\ln (x)=\ln \left(x^{-1}\right)$, so that $B^{(\alpha)}(x)=B_{-\ln }^{(\alpha)}(x), B(x)=B_{-\ln }^{(1)}(x)$ and $B^{e}(x)=B_{-\ln }^{(1 / 2)}(x)$ respectively. We will call $B(x)$ the Brjuno function, which has been mentioned above in Sects. 2 and 3. We have

$B(x)=\sum_{n=0}^{\infty} \beta_{n-1} \ln \left(x_{n}^{-1}\right)=-\ln \left(x_{0}\right)-x_{0} \ln \left(x_{1}\right)+\ldots-x_{0} x_{1} \cdots x_{n-1} \ln \left(x_{n}\right)-\ldots$,

where the $x_{n}$ are obtained from (13) using $\alpha=1$ (Gaussian case), whereas $B^{e}(x)$ is given by the same equation (27) with $x_{n}$ obtained from (13) using $\alpha=\frac{1}{2}$ (continued fraction to the nearest neighbour). Both functions $B(x)$ and $B^{e}(x)$ are 1-periodic, and take value $+\infty$ for $x$ rational. From (25a), the odd part of $B(x)$ is given for $0 \leq x \leq \frac{1}{2}$ by $B_{-}(x)=\frac{1}{2} x\left(\ln \left(x^{-1}-1\right)\right)$, which is continuous (and even Hölder continuous for any exponent $\sigma<1$ ). Moreover, $B^{e}(x)$ is even, and it has been proven [18] that the difference $B^{e}(x)-B^{+}(x)$ is not only bounded, but continuous, and even Hölder continuous for exponent $\frac{1}{2}$. This refines a more general statement [18,22] which says that the differences $B_{\ln }^{(\alpha)}-B(x)$ are bounded over the irrationals.

The numerical computation of $B(x)$ and $B^{e}(x)$ is delicate, due to the instabilities of the continued fraction expansion. However, it is very easy to compute their values when the continued fraction expansion is periodic, that is when $x$ is an irrational quadratic number. This applies to noble numbers, in which case the $x_{n}$ are constant after a certain order.

\section{The Brjuno Series and Diophantine conditions}

A real number is said to be a Brjuno number if and only if $B(x)$ is finite, and we also say that $x$ satisfies the Brjuno diophantine condition. Brjuno numbers are irrationals and real numbers satisfying the classical diophantine conditions (which we recall below) are Brjuno numbers. In [18, we show that for $\frac{1}{2} \leq \alpha \leq 1, B^{(\alpha)}(x)$ is finite if and only if $x$ is a Brjuno number. More precisely, the proof says that for any $\alpha \in\left[0, \frac{1}{2}\right]$, the difference $\left|B^{(\alpha)}(x)-B(x)\right|$ is bounded over irrational values of $x$. We also show that for $\alpha=1$, the 
difference $\left|B(x)-\sum_{n=0}^{\infty} q_{n}^{-1} \ln \left(q_{n+1}\right)\right|$ is bounded over irrational values of $x$, so that we recover the original definition of the Brjuno numbers [23]: $x$ is a Brjuno number if and only if $\sum_{n=0}^{\infty} q_{n}^{-1} \ln \left(q_{n+1}\right)$ is bounded over the irrational. One can see 18,22 that such a definition of the Brjuno numbers does not depend of the particular value of $\alpha$ used to compute the $q_{n}$.

We now report the usual definition [6] of the diophantine conditions : we say that $x$ is an irrational diophantine number of order $\tau \geq 0$ (and we write $x \in \mathrm{C}(\tau)$ ), if there exists $c>0$ such that for any integers $p$ and $q$, such that $q>0$, we have $|x-p / q| \geq c q^{-2-\tau}$. Some classical facts need to be recalled here 24]. First, for any $p$ and $q$ such that $0<q<q_{n+1}$, we have $|q x-p| \geq\left|q_{n} x-p_{n}\right|$, where $p_{n} / q_{n}$ is the Gaussian reduced fraction to $x$. Therefore, in order to have $x \in \mathrm{C}(\tau)$, it is sufficient to check that for any $n>0,\left|x-p_{n} / q_{n}\right| \geq c q_{n}^{-2-\tau}$. Second, Liouville's classical theorem asserts that algebraic numbers of degree $n$ belong to $x \in \mathrm{C}(n-2)$. Moreover Roth's theorem shows that all algebraic numbers belong to $\mathrm{C}(\tau)$, for all $\tau>0$. Finally, for an arbitrary irrational, and any $n>0$, we have $\left(q_{n} q_{n+1}\right)^{-1} \leq$ $\left|x-p_{n} / q_{n}\right| \leq q_{n}^{-2}$. Using (21) for $\alpha=1$, we get an equivalent caracterisation of the diophantine conditions: $x \in \mathrm{C}(\tau)$ if and only if there exists a constant $c>0$ such that $\beta_{n} \geq c \beta_{n-1}^{1+\tau}$ for any $n>0$.

Now we introduce for $\nu>0$, the Brjuno series $B_{\{\nu\}}(x) \equiv B_{x^{-\nu}}(x)$ for the fonctions $f(x)=x^{-\nu}$ (still using $\alpha=1$ ),

$$
B_{\{\nu\}}(x)=\sum_{n=0}^{\infty} \beta_{n-1}(x) x_{n}^{-\nu}=\sum_{n=0}^{\infty} \beta_{n-1}\left(\frac{\beta_{n}}{\beta_{n-1}}\right)^{-\nu}=\sum_{n=0}^{\infty} \beta_{n-1}^{1+\nu}(x) \beta_{n}^{-\nu}(x) .
$$

Using (21) one gets

$$
2^{-\nu} \sum_{n=0}^{\infty} q_{n}^{-1-\nu}\left|q_{n} x-p_{n}\right|^{-\nu} \leq B_{\{\nu\}}(x) \leq \sum_{n=0}^{\infty} q_{n}^{-1-\nu}\left|q_{n} x-p_{n}\right|^{-\nu} .
$$

The series $B_{\{\nu\}}(x)$ converges if and only if the series $\sum_{n=0}^{\infty} q_{n}^{-1-\nu}\left|q_{n} x-p_{n}\right|^{-\nu}$ converges, that is if the series $\sum_{n=0}^{\infty} q_{n}^{-1-2 \nu}\left|x-\left(p_{n} / q_{n}\right)\right|^{-\nu}$ also converges. As a consequence, if $B_{\{\nu\}}(x)<\infty$, then $q_{n}^{-1-2 \nu}\left|x-\left(p_{n} / q_{n}\right)\right|^{-\nu}$ is bounded, and $x \in \mathrm{C}(1 / \nu)$. Conversely, assume $\tau \geq 0$, and $x \in \mathrm{C}(\tau)$, then we have

$$
B_{\{\nu\}}(x) \leq c^{-\nu} \sum_{n=0}^{\infty} q_{n}^{-1+\tau \nu} .
$$

Using bounds in (22), we get the following statement: If $x \in \mathrm{C}(\tau)$, then for any $\nu$ such that $\tau<\nu^{-1}, B_{\{\nu\}}(x)<\infty$. Therefore, there is a relation between the diophantine conditions $\mathrm{C}(\tau)$, and the convergence of the Brjuno series for $f(x)=x^{-\nu}$ : the set of irrationals $x$ such that $B_{\{\nu\}}(x) \equiv B_{x^{-\nu}}(x)$ is bounded, is contained in $\mathrm{C}(1 / \nu)$, and contains $\mathrm{C}(-\varepsilon+1 / \nu)$, for any $0<\varepsilon \leq 1 / \nu$. In some sense, the Brjuno conditions is related to the limiting case $\nu=0$, 
and in particular, $x \in \mathrm{C}(\tau)$ for $\tau>0$ implies $B(x)<\infty$, that is $x$ is a Brjuno number. Using a more general function $f$, positive on $(0,1)$, and monotoneously decreasing in the vicinity of zero, we can introduce a wide family of conditions $B_{f}(x)<+\infty$. The diophantine conditions obtained will be mainly governed by the singular behavior of $f$ around zero. A power law behaviour would simulate the usual conditions, whereas a logarithmic behaviour would generate a condition similar to the Brjuno condition. Other interesting examples would be obtained by taking functions $f$ of the form $x^{-\nu}|\log (x)|^{\mu}, x^{-\nu}|\log (x)|^{\mu}|\log (|\log x|)|^{\sigma}$, and so one.

\section{The Brjuno Operator}

We will introduce now some functional analysis in order to solve Equations $(25 \mathrm{a}-\mathrm{c})$. For fixed $\frac{1}{2} \leq \alpha \leq 1$, let us consider the operator $T_{(\alpha)}$, acting on locally Lebesgue integrable functions $f$ on the real line, which verify

$$
\begin{aligned}
& f(x)=f(x+1) \text { for almost every } x \in \mathbb{R}, \\
& f(x)=f(-x) \text { for almost every } x \in(0,1-\alpha) .
\end{aligned}
$$

The operator is defined by

$$
\left(T_{(\alpha)} f\right)(x)=x f\left(\frac{1}{x}\right), \text { if } x \in(0, \alpha) .
$$

It is understood that the function $T_{(\alpha)} f$ is completed outside $(0, \alpha)$ by imposing on $T_{(\alpha)} f$ the same parity and periodicity conditions which are expressed for $f$ in the above equations $(31 \mathrm{a}-\mathrm{b})$. The functional equations $(25 \mathrm{a}-\mathrm{c})$ can then be written in the form

$$
\left(1-T_{(\alpha)}\right) B_{f}^{(\alpha)}=f .
$$

This suggest to study the operator $T_{(\alpha)}$ on the Banach spaces

$$
X_{\alpha, p}=\left\{f: \mathbb{R} \rightarrow \mathbb{R} \mid f \text { verifies }(31 \mathrm{a}-\mathrm{b}), \quad f \in L^{p}(0, \alpha)\right\}
$$

endowed with the norm of $L^{p}(0, \alpha)$, namely

$$
\|f\|_{\alpha, p}=\left(\int_{0}^{\alpha}|f(x)|^{p} d x\right)^{1 / p}
$$

for $p \in[1, \infty]$. Note that one could also use $L^{p}(0,1)$, instead of $L^{p}(0, \alpha)$, and that if $p<p^{\prime}$ one has the obvious inclusion $X_{\alpha, p^{\prime}} \subset X_{\alpha, p}$. If $\left(1-T_{(\alpha)}\right)$ is invertible in the considered space, then $(25 \mathrm{a}-\mathrm{c})$ have a unique solution for $B_{f}^{(\alpha)}$, provided that the $f$ in the right hand side of $(25 \mathrm{~b})$ also belongs to the space. The invertibility property is given by the following theorem, which states in particular that the spectral radius of $T_{(\alpha)}$ is strictly smaller than 1. 
Theorem. $T_{(\alpha)}$ is a linear bounded operator from $X_{\alpha, p}$ into itself for all $\alpha \in\left[\frac{1}{2}, 1\right]$ and for all $p \in[1, \infty]$. Its spectral radius on $X_{\alpha, p}$ is bounded by the constant $\lambda(\alpha)$ of Equation (22), and therefore $1-T_{(\alpha)}$ is invertible.

For the proof, see [18]. We will just observe here that the result is immediate in the $p=\infty$ case. Indeed,

$$
\left(T_{(\alpha)}^{n} f\right)(x)=\beta_{n-1}(x) f\left(x_{n}\right)=\beta_{n-1}(x)\left(f \circ A_{\alpha}^{n}\right)(x),
$$

where the map $A_{\alpha}$ is defined above (see $\left.(16 \mathrm{a}-\mathrm{b})\right)$. Therefore

$$
\left\|T_{(\alpha)}^{n} f\right\|_{\alpha, \infty} \leq \sup _{x}\left(\beta_{n-1}(x)\right)\|f\|_{\alpha, p} \leq c \lambda(\alpha)^{n-1}\|f\|_{\alpha, p}
$$

and one gets the theorem (for $p$ infinite) by taking the $1 / n$-th root of both sides. For the other values of $p$, it is convenient to make use of the measure which is invariant under transformation by the map $A_{\alpha}$, instead of the Lebesgue measure. An immediate consequence of the theorem, is that if we take $f(x)=\ln (x)$, for $0<x<\alpha$, then $f \in X_{\alpha, p}$, for all finite $p$ and therefore we also have $B^{(\alpha)} \in X_{\alpha, p}$ for all finite $p$.

However, we have a stronger property in the $\alpha=\frac{1}{2}$ case. Here, we set again $T_{e} \equiv T_{(1 / 2)}$. In this case, the logarithm belongs also to the set $X_{*} \subset$ $X_{\alpha=1 / 2, p=1}$, made of even, periodic (with period 1) functions belonging to the so-called "BMO-space". In this space, $f$ has bounded mean oscillation, more precisely the following semi-norm $\|f\|_{*}$ is bounded, with

$$
\|f\|_{*}=\sup _{I} \frac{1}{|I|} \int_{I}\left|f-f_{I}\right| d x
$$

where the mean value of $f$ over $I$ is $f_{I}=|I|^{-1} \int_{I}\left|f-f_{I}\right| d x$, and the sup is taken over all possible intervals $I$ with length $|I|$ smaller than one. The BMO space has remarkable properties. First it is contained in all $L_{p}$ spaces for $p$ finite, and it contains the $L_{\infty}$ space, second it is the space adequate to describe functions having singular behaviour not worse than logarithmic, but around every point in a dense set of the real line, and third, it has remarkable properties connected to the harmonic conjugacy transformation [25, 18$]$.

In [18,26], we have shown that $1-T_{e} \equiv 1-T_{(1 / 2)}$ is invertible in $X_{*}$, and therefore that $B^{e} \equiv B^{(1 / 2)} \in X_{*}$. Since $\left|B^{\alpha}-B^{e}\right|$ is bounded for $\frac{1}{2} \leq \alpha \leq 1$, we have the unexpected consequence that all $B^{(\alpha)}$ for $\frac{1}{2} \leq \alpha \leq 1$, also have the Bounded Mean Oscillation property, although it cannot be shown directly through the properties of $1-T_{(\alpha)}$.

The BMO property obtained for the Brjuno function in the real case, was one of our motivations to consider the complexification procedure which we will describe in the last Section of this paper [27].

\section{Application to Hölder-continuous Functions}

In this Section, we will consider only the case $\alpha=\frac{1}{2}$. In this case the map $A_{\alpha} \equiv A_{1 / 2}$ is continuous on the interval $\left(0, \frac{1}{2}\right]$. The functional equation for 
the Brjuno function $B^{e}$ for $\alpha=1 / 2$ is

$$
\left[\left(1-T_{e}\right) B^{e}\right](x)=-\log x
$$

for all $x \in(0,1 / 2)$, complemented with the condition that $B^{e}$ is even and periodic. We suppose that the right hand side of this equation is pertubed, by an additional term $f$, which is less singular than the logarithmic function, and we want to study the singular properties of the perturbed solution. Since the equation is linear, we only need to consider the action on $f$ of $T_{e}$ and $\left(1-T_{e}\right)^{-1}$, which we will conveniently recall the Brjuno operator $\mathbf{B}_{\mathbf{e}}$. We will consider even and periodic functions $f$ which are continuous. It is sufficient to know the value of $f$ on $[0,1 / 2]$, so we assume $f \in C_{[0,1 / 2]}^{0}$. One can check that $T f$ is also continuous provided we set $T f(0)=0$. We need now the usual Hölder's type semi-norms for continuous functions : let $f \in C_{[0,1 / 2]}^{0}$, then we define the Hölder's $\gamma$-norm as

$$
|f|_{\gamma}=\sup _{0 \leq x<y \leq 1 / 2} \frac{|f(x)-f(y)|}{|x-y|^{\gamma}},
$$

with $0<\gamma \leq 1$. This is a seminorm since it vanishes on constant functions, so that we introduce the norm:

$$
\|f\|_{\gamma}=A|f|_{\gamma}+B|f|_{\infty}
$$

where $|f|_{\infty}$ is the $L_{\infty}$ norm of $f$, and $A$ and $B$ are positive constants which we can choose arbitrarily, provided that they do not vanish. We say that $f \in C^{\gamma}$, if $f \in C_{[0,1 / 2]}^{0}$ and $|f|_{\gamma}$ is finite. We now have:

Proposition. $T_{e}$ is a bounded operator in $C^{\gamma}$, for the norm $\|f\|_{\gamma}$, when $0<\gamma \leq 1 / 2$, provided $B / A$ is large enough: if $B / A>\left(2^{\gamma}-2^{-\gamma}\right)^{-1}$, the norm of $T_{e}$ corresponding to the norm (41) satisfies $\left\|T_{e}\right\|_{\gamma} \leq 2^{(2 \gamma-1)} \leq 1$. Therefore for $0<\gamma<1 / 2, T_{e}$ is a contraction, and $1-T_{e}$ is invertible.

We need the following Lemma

Lemma. Let $0<y<x \leq 1 / 2$, and define $x_{1}$ and $y_{1}$ by the following conditions

$$
y=\frac{1}{n+y_{1}} \quad, \quad x=\frac{1}{m+x_{1}},
$$

with $n \geq 2$ and $m \geq 2$, and $-1 / 2 \leq x_{1}<1 / 2$ and $-1 / 2 \leq y_{1}<1 / 2$, then we have

$$
|| x_{1}|-| y_{1}|| \leq \frac{|x-y|}{|x||y|} .
$$

Proof of the Lemma. Since $y<x$, we have $n-m>x_{1}-y_{1}>-1$. therefore $n \geq m$. Let $n-m=p \geq 0$. We have $x-y=x y\left(p+y_{1}-x_{1}\right)$. So that we need to prove ||$x_{1}|-| y_{1}|| \leq\left|p+y_{1}-x_{1}\right|$. This is obvious when $p=0$. We always have ||$x_{1}|-| y_{1}|| \leq 1 / 2$, so the required inequality also holds when $p \geq 2$. In the remaining case $p=1$, we set $\eta=\operatorname{sign}\left(y_{1}\right)$ and $\epsilon=\operatorname{sign}\left(x_{1}\right)$, and we need 
to check that ||$x_{1}|-| y_{1}|| \leq|1+\eta| y_{1}|-\epsilon| x_{1}||$. Still because the left hand side is smaller or equal to $1 / 2$, this last inequality is not obvious only when $\eta=-1$ and $\epsilon=+1$. It therefore remains to show that ||$x_{1}|-| y_{1}|| \leq|1-| y_{1}|-| x_{1}||$. Setting $u=1 / 2-\left|x_{1}\right|$ and $v=1 / 2-\left|y_{1}\right|$, the last inequality is equivalent to $|1-v / u| \leq|1+v / u|$, which is readily checked since $u / v$ is real and nonnegative.

Proof of the Proposition. Let $0<y<x \leq 1 / 2$, and $x_{1}$ and $y_{1}$ as in the preceding lemma. We have

$$
\begin{aligned}
\left|T_{e} f(x)-T_{e} f(y)\right| & =|x f(1 / x)-y f(1 / y)|=\left|x f\left(\left|x_{1}\right|\right)-y f\left(\left|y_{1}\right|\right)\right| \\
& \leq|x-y|\left|f\left(\left|x_{1}\right|\right)\right|+|y|\left|f\left(\left|x_{1}\right|\right)-f\left(\left|y_{1}\right|\right)\right| \\
& \leq|x-y||f|_{\infty}+|y||f|_{\gamma}|| x_{1}|-| y_{1}||^{\gamma} \\
& \leq|x-y||f|_{\infty}+|f|_{\gamma} \frac{|x-y|^{\gamma}}{|x|^{\gamma}|y|^{\gamma-1}}
\end{aligned}
$$

where we have used $f \in C^{\gamma}$, and the Lemma. Therefore

$$
\begin{aligned}
\frac{\left|T_{e} f(x)-T_{e} f(y)\right|}{|x-y|^{\gamma}} & \leq|x-y|^{1-\gamma}|f|_{\infty}+\left(\frac{|y|}{|x|}\right)^{\gamma}|y|^{1-2 \gamma}|f|_{\gamma} \\
& \leq(1 / 2)^{1-\gamma}|f|_{\infty}+(1 / 2)^{1-2 \gamma}|f|_{\gamma},
\end{aligned}
$$

since $0<y<x \leq 1 / 2$, and $\gamma \leq 1 / 2$. For $y=0$, the right hand side can be replaced by its first term $(1 / 2)^{1-\gamma}|f|_{\infty}$, and the above inequality extends to the case where $y$ vanishes, so that

$$
\left|T_{e} f\right|_{\gamma} \leq K_{\gamma}(f)=2^{\gamma-1}|f|_{\infty}+2^{2 \gamma-1}|f|_{\gamma} .
$$

For the norm, we get

$$
\begin{aligned}
\left\|T_{e} f\right\|_{\gamma} & =A\left|T_{e} f\right|_{\gamma}+B\left|T_{e} f\right|_{\infty} \\
& \leq 2^{2 \gamma-1}\left[A|f|_{\gamma}+\left(2^{-2 \gamma} B+2^{-\gamma} A\right)|f|_{\infty}\right], \\
& \leq 2^{(2 \gamma-1)}\|f\|_{\gamma},
\end{aligned}
$$

provided $2^{-2 \gamma} B+2^{-\gamma} A \leq B$, that is $A / B \leq 2^{\gamma}-2^{-\gamma}$ which completes the proof. The above proposition has two obvious consequences

- Since $C^{\gamma} \subset C^{\gamma^{\prime}}$ whenever $\gamma^{\prime} \leq \gamma$, we have

$$
\begin{aligned}
& f \in C^{\gamma} \quad \text { and } \quad \gamma \geq 1 / 2 \quad \Longrightarrow \quad T_{e} f \in C^{1 / 2} \\
& f \in C^{\gamma} \quad \text { and } \quad \gamma \geq \gamma_{0} \quad, \quad \gamma_{0} \leq 1 / 2 \quad \Longrightarrow \quad T_{e} f \in C^{\gamma_{0}}
\end{aligned}
$$

- When $\gamma<1 / 2, T_{e}$ is a contraction on $C^{\gamma}$. Therefore $1-T_{e}$ is invertible and $\mathbf{B}_{\mathbf{e}}=\sum_{0}^{\infty}\left(T_{e}\right)^{n}=\left(1-T_{e}\right)^{-1}$ preserves $C^{\gamma}$, and we have

$$
\begin{aligned}
& f \in C^{\gamma} \text { and } \quad 0<\gamma<1 / 2 \quad \Longrightarrow \quad \mathbf{B}_{\mathbf{e}} f \in C^{\gamma} \\
& f \in C^{1 / 2} \quad \Longrightarrow \quad \mathbf{B}_{\mathbf{e}} f \in C^{\gamma} \quad, \quad \forall \gamma \text { such that } 0<\gamma<1 / 2 .
\end{aligned}
$$


We have reproduced here the proof of [26, because it is essentially elementary. In fact we have a slightly better result for $\mathbf{B}_{e}$ than for $T_{e}$, as shown in the next proposition, which shows that the $C^{1 / 2}$ property is effectively reached. Its proof 18,26] is too difficult to be reproduced here. We have Proposition. If $f \in C^{\gamma}$, and $\gamma>1 / 2$, then $\mathbf{B}_{e} f \in C^{1 / 2}$.

The Brjuno function $B^{e}$ which we have studied in the previous section is nothing else than $\mathbf{B}_{e} \ell$, where $\ell$ is equal to minus the logarithmic fonction restricted to $[0,1 / 2]$. When made even and periodic, this function is not continuous. Suppose that we perturb $\ell$ by a function with enough regularity properties (for example $C^{1}$ or $C^{1 / 2+\epsilon}$ ), the change in $\mathbf{B}_{e} \ell$ will be continuous and even in $C^{1 / 2}$, that is Hölder- $\frac{1}{2}$ continuous. In this sense, the 'most singular part' of the Brjuno function is stable or 'universal', roughly speaking modulo Hölder- $\frac{1}{2}$ continuous contributions. As noticed at the end of Section 5 above, we can use either $B^{e} \equiv B^{(1 / 2)}$ or $B \equiv B^{(1)}$ since a similar argument starting from $(26 \mathrm{a}-\mathrm{c})$ shows that their difference is also Hölder- $\frac{1}{2}$ continuous 18$]$.

This provides a frame to understand the properties of the critical constants $K$ of the Sections 2 and 3 above. We assume that the singularity comes from the renormalisation equation $\left(1-T^{e}\right) K=\ell+f$, and not from additional singular behaviour coming from $f$ in the right-hand side. If it would exist, such an additional singular behaviour would require a further physical interpretation. This argument, which is usual in the renormalisation analysis of singularities, was one of the motivation for our previous work [18. The renormalisation equation allows naturally to conjecture that the difference between the Brjuno function $B^{e}$, and the logarithm of the various critical constants (multiplied by a suitable coefficient), is continuous and even Hölder- $\frac{1}{2}$ continuous. As an example, we conjecture that the ratio of $e^{-B}$ and the radius of the Siegel disk of the quadratic polynomial, is an Hölder- $\frac{1}{2}$ continuous function of the rotation number. These conjectures are in agreement with the numerical results [16]10.

\section{The Complexification of the Brjuno Function}

We consider here the case $\alpha=1$, and we want to associate to the function $f$ in $X_{1,2}$, a function $\Phi$, holomorphic in the upper half plane, such that $\operatorname{Im} \Phi \rightarrow B_{f}$ when z goes to the real axis. Since $\operatorname{Re} \Phi$ is associated to the harmonic conjugate of $f$, we expect to find better boundedness properties when $f$ has the BMO property. We will here describe our procedure, and report the results [27].

We associate to $f$ a function $F(z)$ holomorphic in $\mathbb{C} \backslash[0,1]$, and vanishing at infinity, as follows

$$
F(z)=\frac{i}{\pi} \int_{o}^{1} \frac{f(x)}{x-z} d x .
$$

For $\mathrm{x}$ real, we have $\operatorname{Im} F(x \pm i \varepsilon)= \pm f(x)$ for $x \in[0,1]$, and $\operatorname{Im} F(x)=0$ for $x \notin[0,1]$. We will be particularly interested in the case $f(z)=\ln (z)$, in 
which case we get $F(z)=-\pi^{-1} \mathrm{Li}_{2}(1 / z)$, where $\mathrm{Li}_{2}$ is the classical dilogaritm function [28]. Now we set

$$
\Phi(z)=\lim _{N \rightarrow \infty} \sum_{-N}^{+N} F(z+n)
$$

and we get a function $\Phi$ holomorphic in the upper-half plane $\mathbb{H}_{+}$, periodic with a real period equal to one, and such that for $\mathrm{x}$ real, $\operatorname{Im} F(x \pm i \varepsilon)= \pm f(x)$. In fact the previous equation defines a pair on functions $\Phi_{ \pm}$, respectively holomorphic in the upper or lower half plane $\mathbb{H}_{ \pm}$, so that the natural frame in which our procedure takes place is the frame of complex hyperfunctions, which we will not consider here [27].

We consider now the action of $T$, with $(T f)(x)=x f(1 / x)$ if $0 \leq x<1$, $f$ and $T f$ being complemented using periodicity. Using the above correspondence $f \mapsto T f$, a correspondence $F \mapsto T F$ is induced on holomorphic functions in $\mathbb{C} \backslash[0,1]$, vanishing at infinity. We get

$$
(T F)(z)=-z \sum_{m=1}^{\infty}\left(F\left(\frac{1}{z}-m\right)-F(-m)\right)+\sum_{m=1}^{\infty} F^{\prime}(-m) .
$$

In fact, $T F$ is essentially $-z \sum_{m=1}^{\infty} F\left(z^{-1}-m\right)$, up to an affine additive correction, which could be determined by the vanishing condition at infinity.

If $f$ is associated to $F$ as above, the solution $B_{f}(x)$ (for $\left.\alpha=1\right)$ of $(25 \mathrm{a}-\mathrm{c}$ ) is associated to the series

$$
\mathcal{B}_{f}(z)=\sum_{\mathbb{Z}} \sum_{m=0}^{\infty}\left(T^{n} F(z)\right)
$$

where we use the notation $\sum_{\mathbb{Z}} F$ for $\Sigma_{n=-\infty}^{+\infty} F(z+n)$ understood as the symmetric summation (51) to insure convergence.

It is now interesting to display the link between (53) and the modular group $G L(2, \mathbb{Z})$. Let $g=\left(\begin{array}{ll}a & b \\ c & d\end{array}\right) \in G L(2, \mathbb{Z})$, which mean $a, b, c, d \in \mathbb{Z}$, $\varepsilon_{g}=a d-b c= \pm 1$. To $g$ we associate the following group action on functions holomorphic on $\mathbb{C} \backslash[0,1]$, that is $g \mapsto L_{g} F$, with

$$
\left(L_{g} F\right)(z)=(a-c z)\left\{F\left(\frac{d z-b}{a-c z}\right)-F\left(-\frac{d}{c}\right)\right\}-\frac{\varepsilon_{g}}{c} F^{\prime}\left(-\frac{d}{c}\right) .
$$

Let $\mathcal{M}_{+} \subset G L(2, \mathbb{Z})$ be the multiplicative monoid generated by the unit matrix, and the set of matrices $\left(\begin{array}{cc}0 & 1 \\ 1 & m\end{array}\right)$, for $m \geq 1$ integer. The monoid $\mathcal{M}_{+} \subset G L(2, \mathbb{Z})$ can also be defined as the set of matrices including identity and the matrices $\left(\begin{array}{ll}a & b \\ c & d\end{array}\right) \in G L(2, \mathbb{Z})$ such that first, $d \geq c \geq a \geq 0$, and 
second, $d \geq b \geq a \geq 0$. In $G L(2, \mathbb{Z})$ there is a unique product decomposition, namely $\forall g \in G L(2, \mathbb{Z})$ there exist a unique set of three matrices $k, m$ and $h$, with $g=k m h$, and $m \in \mathcal{M}^{+}, k \in Z$, where $\mathrm{Z}$ is the translation subgroup of matrices $\left(\begin{array}{ll}1 & n \\ 0 & 1\end{array}\right), n \in \mathbb{Z}$, and $h \in H$, where $h$ is the order eight sugroup of $G L(2, \mathbb{Z})$ made of the matrices $\left(\begin{array}{cc}\varepsilon & 0 \\ 0 & \varepsilon^{\prime}\end{array}\right)$, and $\left(\begin{array}{cc}0 & \varepsilon \\ \varepsilon^{\prime} & 0\end{array}\right)$, with $\varepsilon= \pm 1$ and $\varepsilon^{\prime}= \pm 1$.

Now (53) is rewritten as

$$
\mathcal{B}_{f}(z)=\sum_{k \in Z} \sum_{g \in \mathcal{M}^{+}}\left(L_{(h g)} F\right)(z)
$$

The double sum over $g$ and $k$ amounts to a sum over a part of the full modular group (here one over eight). The contribution over the seven other possible parts would be $-\mathcal{B}_{f}(z), \pm \mathcal{B}_{f}\left(z^{-1}\right), \pm \mathcal{B}_{f}(-z)$, and $\mathcal{B}_{f}\left(-z^{-1}\right)$.

We will now summarize the results:

i) The sums in (55) converge in the open upper half plane as long as $f$ is in $L_{1}(0,1)$ which insures that $F$ is holomorphic in $\mathbb{C} \backslash[0,1]$, and vanishes at infinity.

ii) When $f$ is in $L_{p}(0,1), p$ finite, then $\mathcal{B}_{f}$ is in the Hardy $\mathbb{H}_{p}$ space.

iii) If $f$ is such that $F$ has bounded real part, then the same holds for $\mathcal{B}_{f}$.

iv) For $f(x)=\ln (x)$, and $F(z)=-\pi^{-1} \operatorname{Li}_{2}(1 / z)$, we get the complexified Brjuno function, $\mathcal{B}$, holomorphic in the upper half plane, vanishing at $+i \infty$. When $z$ goes to a real number in a non-tangential way, we have the following limits when $\varepsilon>0$ goes to zero : the real part $\operatorname{Re} \mathcal{B}(x+i \varepsilon)$ has a bounded limit for any real $x$. This limit is continuous at all irrationals and has a decreasing jump of $\pi / q$ at each rational $p / q$. When $x$ is a Brjuno number, $\operatorname{Im} \mathcal{B}(x+i \varepsilon)$ goes to the Brjuno function $B(x)$.

The limit properties of the complex Brjuno function on the real axis are characteristic of functions $f$ having a logarithmic singularity aroud zero. Although the boundedness of the real part reminds the BMO property of the real Brjuno function, it is in fact a stronger property. This is one more remarkable feature of this function. We are convinced that the interpretation of the properties of the Brjuno function in terms of the modular group is promising. On the other hand, we can hope to find an interpretation of the complexified version of rotation numbers analogous with the usual interpretation of complex frequencies in terms of damped oscillations, but this is another story.

Acknowledgements. The first author thanks the CNRS and the organizors of the École Thematique held at La Chapelle des Bois, with special thanks for Michel Planat. This work begun during a visit of the second author at the S.Ph.T.-CEA Saclay and at the Dept. of Mathematics of Orsay during the academic year 1993-1994. This research has been supported by the CNR, 
CNRS, INFN, MURST and a EEC grant. We thank J.-C. Yoccoz for his constant help and warm encouragements, and also for allowing us to report here results obtained in collaboration with him.

\section{References}

1. Chirikov, B. (1979) A universal instability of many- dimensional oscillator systems. Phys. Reports, 52, 263-279

2. Escande, D. (1985) Stochasticity in classical Hamiltonian systems: universal aspects. Phys. Reports, 121, 165-261

3. Greene, J. M. (1979) A method for determining a stochastic transition. J. Math. Phys. 20, 1183-1201

4. Aubry S. and Le Daeron, P. (1983) The discrete Frenkel-Kontorova model and its extensions. Physica 8D, 381-422

5. Mather J. N. (1984) Non existence of invariant circles. Ergod. Theor. and Dynam. Sys. 4, 301-309

6. Yoccoz J.-C. (1992) An introduction to small divisors problems, in: From Number Theory to Physics, Waldschmidt M., Moussa P., Luck J.-M., and Itzykson C. editors, Springer-Verlag, Berlin, pp. 659-679

7. Berretti A. and Gentile G. (1998) Scaling properties of the radius of convergence of the Lindstedt series : the standard map. University of Roma, Italy, preprint

8. Berretti A. and Gentile G. (1998) Bryuno function and the standard map. University of Roma, Italy, preprint

9. Yoccoz J.-C. (1995) Théorème de Siegel, nombres de Bruno et polynômes quadratiques. Astérisque, 231, 3-88, (appeared first as a preprint in 1987).

10. Marmi S. and Stark J. (1992) On the standard map critical function. Nonlinearity 5, 743-761

11. Carletti T. and Laskar J. (1999) Scaling law in the standard map critical function, interpolating hamiltonian and frequency analysis. (Preprint, Bureau des Longitudes, Paris, in preparation)

12. Treshev D. and Zubelevitch O. (1998) Invariant tori in Hamiltonian systems with two degrees of freedom in a neighborhood of a resonance. Regular and Chaotic dynamics, 3, 73-81

13. Gelfreich G. V. (1999) A proof of exponentially small transversality of the separatrices for the standard map. Commun. Math. Phys. 201, 155-216

14. Davie A. M. (1995) Renormalisation for analytic area preserving maps. University of Edinburgh preprint

15. Davie A. M. (1994) the critical function for the semistandard map. Nonlinearity 7, 219-229

16. Marmi S. (1990) Critical functions for complex analytic maps function. J. Phys. A: Math.Gen. 23, 3447-3474

17. Perez-Marco R. (1992) Solution complète du problème de Siegel de linéarisation d'une application holomorphe autour d'un point fixe. Séminaire Bourbaki nr.753, Astérisque, 206, 273-310

18. Marmi S., Moussa P., and Yoccoz J.-C. (1997) The Brjuno function and their regularity properties. Commun. Math. Phys. 186, 265-293

19. Buric N., Percival I. C., and Vivaldi F. (1990) Critical function and modular smoothing, Nonlinearity 3, 21-37 
20. MacKay R. S. (1988) Exact results for an approximate renormalisation scheme and some predictions for the breakup of invariant tori, Physica 33D, 240-265, and Erratum (1989) Physica 36D, 358-265

21. Schweiger F. (1995) Ergodic theory of fibered systems and metric number thory, Clarendon Press, Oxford,

22. Moussa P., Cassa A., and Marmi S. (1999) Continued fractions and Brjuno functions, J. Comput. Appl. Math. 105 403-415

23. Brjuno. A. D. (1971) Analytical form of differential equations, Trans. Moscow Math. Soc. 25 131-288, and, (1972), 26 199-239

24. Hardy G. H., and Wright E. M. (1938) An introduction to the theory of numbers, Clarendon Press, Oxford, chapter 11, fifth edition 1979

25. Garnett J. B. (1981) Bounded Analytic functions, Academic Press, New York.

26. Marmi S., Moussa P., and Yoccoz J.-C. (1995) Développements en fractions continues, fonctions de Brjuno et espaces BMO, CEA/Saclay, Note CEA-N-2788

27. Marmi S., Moussa P., and Yoccoz J.-C. (1999) Complex Brjuno functions, Preprint SPhT/CEA Saclay T99/066, 71 p.

28. Lewin L, (1981) Polylogarithms and Associated Functions, Elsevier North Holland, New York. 